\title{
Meiotic progression of isolated mouse spermatocytes under simulated microgravity
}

\author{
Silvia Di Agostino, Flavia Botti, Anna Di Carlo, Claudio Sette and Raffaele Geremia \\ Dipartimento di Sanità Pubblica e Biologia Cellulare, Università di Roma 'Tor Vergata', Via Montpellier 1, 00133, \\ Rome, Italy
}

Correspondence should be addressed to R Geremia; Email: geremia@med.uniroma2.it

\begin{abstract}
Progression through the prophase of the first meiotic division can be obtained in culture by treatment of mouse spermatocytes with the serine/threonine phosphatase inhibitor okadaic acid. Chromosome condensation during this G2/M transition involves the activation of the MAPK pathway, which causes the activation of Nek2 and the phosphorylation of the chromatin architectural protein Hmga2. In an effort to set up conditions to allow a spontaneous progression of mouse spermatocytes through meiosis, we have investigated the cell-cycle features of these cells cultured for $24 \mathrm{~h}$ with a rotary cell culture system in a humidified atmosphere in a thermostatic incubator to simulate a microgravity environment. Morphological analysis of nuclear squashes indicated a 2-fold increase in late-pachytene spermatocytes with highly condensed chromosomes, and a contemporaneous decrease of mid-pachytene cells with less condensed chromatin. Microgravity induced a 2-fold activation of the cyclinB-cdc2 complex, confirming at the molecular level that cell-cycle progression had occurred. Moreover, using immunokinase assays with specific substrates we have demonstrated that the meiotic progression obtained under microgravity conditions is accompanied by activation of the Erk1/p90Rsk2 pathway. These data indicated that activation of the MAPK pathway correlates with chromatin condensation even under conditions in which meiotic progression occurs spontaneously and is not induced by a drug. We suggest that culture under microgravity conditions might help to release the block that inhibits isolated spermatocytes from progressing through prophase at unit gravity, and to study the physiological events of germ cell differentiation in vitro.

Reproduction (2004) 128 25-32
\end{abstract}

\section{Introduction}

Meiosis is characterized by a single round of DNA replication followed by two cell divisions, in which a single diploid cell gives rise to four haploid cells. The main events that differentiate meiosis from mitosis occur during the prophase of the first meiotic division. In the mouse, this stage lasts several days during which homologous chromosomes anneal along the synaptonemal complex and genetic recombination between them takes place (Roeder 1997, Petronczki et al. 2003). At pachytene, the crossing-over is terminated and chromatin is condensed progressively to yield the meiotic chromosomes. Another peculiar feature of the meiotic cycle is the lack of the G1-S phases in the interphase between the two cell divisions; in fact, chromatin is not fully decondensed and DNA is not duplicated, ensuring the reduction of the DNA content in the resulting daughter cells (for reviews see Roeder 1997, Maller et al. 2001). Most of the knowledge available about the control of the meiotic cell cycle in vertebrates was obtained using the maturing Xenopus laevis oocyte as a model system (Sagata 1997, Abrieu et al. 2001). This oocyte is naturally arrested in prophase (diplotene) of the first meiotic prophase; stimulation with progesterone triggers cell-cycle progression, extrusion of the first polar body and a new arrest at metaphase of the second meiotic division. At the molecular level, progesterone leads to the expression of the protein kinase Mos and the consequent activation of the MAPK cascade (Sagata et al. 1988, 1989), and to the activation of the polo-like kinase and the consequent activation of the cyclinB/Cdc2 complex (MPF) (Qian et al. 2001). Although all these events are required for the correct completion of meiosis, the interplay between these kinases has not been completely elucidated. Initially, translation of Mos was considered to be the trigger of meiotic resumption (Sagata et al. 1989). Mos acts as an MAPKK to trigger activation of Erk1/2 and of their effector, the serine/threonine kinase p90Rsk2. This kinase was shown to relieve the inhibition of MPF and cause cell-cycle resumption through phosphorylation and inactivation of the negative regulator 
Myt1 (Palmer et al. 1998). However, more recent data, also supported by the phenotype of mice ablated for the mos gene, seem to rule out the requirement of the Mos/ MAPK/p90Rsk2 pathway for MPF activation and germinal vesicle breakdown (GVBD), and to restrict its function to the suppression of the interphase between the two meiotic divisions, to the assembly of the meiotic spindle and to the regulation of the anaphase-promoting complex that allows metaphase arrest in the second division (Gross et al. 2000, Dupre et al. 2002).

As anticipated above, mouse knock-out models for mos also suggest that the MAPK pathway is not required for MPF activation and GVBD at the resumption of meiosis, but rather for spindle formation during the second division and for the metaphase II arrest (Colledge et al. 1994, Hashimoto et al. 1994, Verlhac et al. 1996, 2000). Because of the model system used (i.e. oocyte arrested in diplotene/dictyate stage), not much information on the role of the MAPK pathway in earlier meiotic stages was provided by these studies. In the past few years, however, biochemical analysis of earlier meiotic transitions of mouse spermatocytes was made possible by a synchronization protocol exploited by Wiltshire et al. (1995) which allows pachytene spermatocytes to enter metaphase within $4-6 \mathrm{~h}$ under stimulation in vitro with the serine/ threonine kinase inhibitor okadaic acid (OA). Although artificially induced, metaphase chromosomes obtained by this treatment were described as normal bivalents in which crossing-over was completed, the synaptonemal complex had dissolved and chiasmata were present (Wiltshire et al. 1995), indicating that the process of chromatin condensation had occurred correctly. Using this culture protocol, we have recently suggested that the MAPK pathway plays a role in chromosome condensation during the pachytene-to-metaphase transition of primary spermatocytes (Sette et al. 1999, Di Agostino et al. 2002). Meiotic progression was accompanied by activation of MPF and MAPKs (Sette et al. 1999). Moreover, we have observed that activation of the MAPK/p90Rsk2 pathway was required for the efficient condensation of the chromatin into metaphase chromosomes (Sette et al. 1999, Di Agostino et al. 2002). Once activated, p90Rsk2 interacted with and activated Nek2 (Di Agostino et al. 2002, 2004), a kinase associated with condensing chromosomes during the prophase of the first division (Rhee \& Wolgemuth 1997) and which binds to and phosphorylates the DNA architectural protein Hmga2 causing its release from the condensing chromatin (Di Agostino et al. 2004).

Since most of our studies were performed using OA as a trigger of meiotic progression, and because this drug causes the activation of multiple serine/threonine kinase pathways (Sassa et al. 1989), we were interested in determining a more physiological system to study male meiotic progression in culture. In this study, we have examined some morphological and biochemical events on pachytene spermatocytes cultured in rotating-wall vessels that provide a simulated microgravity condition. This novel in vitro culture technology has been used to improve tissue culture conditions: it was demonstrated that fibroblasts or carcinoma tissues cultured under a simulated microgravity condition show a greater number of mitotic cells and a lower number of apoptotic cells than control cells cultured under gravity condition (Ingram et al. 1997, Nakamura et al. 2002). At the molecular level, it was also shown that the microgravity culture system leads to upregulation of the expression of mitotic genes in proliferating cells (Nakamura et al. 2002). Our results on mouse spermatocytes have shown that microgravity culture conditions promote spontaneous meiotic progression, as monitored by the significant increase of chromatin condensation and by molecular parameters such as activation of MAPKs, p90Rsk2 kinase and MPF. These data suggest that microgravity influences the meiotic progression of mouse spermatocytes and that activation of the MAPK pathway occurs spontaneously during the pachytene stage of male meiosis even in the absence of drug treatment.

\section{Materials and Methods \\ Cell isolation, culture and treatments}

Testes from 18- to 20-day-old CD1 mice (Charles River, Milan, Italy) were used to obtain pachytene spermatocytes by elutriation techniques as previously described (Meistrich 1977). After elutriation, pachytene spermatocytes were cultured for $24 \mathrm{~h}$ in minimum essential medium, supplemented with $0.5 \%$ bovine serum albumin (BSA), $1 \mathrm{mM}$ sodium pyruvate and $2 \mathrm{mM}$ sodium lactate, at a density of $10^{6}$ cells $/ \mathrm{ml}$ at $32{ }^{\circ} \mathrm{C}$ in a humidified atmosphere containing $95 \%$ air and $5 \% \mathrm{CO}_{2}$. Cultures were performed both in conventional tissue culture dishes maintained in unit gravity, and in a rotary cell culture system (RCCS) (RCCS-4D; Synthecon Inc., El Rio, Houston, TX, USA) under a rotation rate of 10 revolutions/min which provides a simulated microgravity condition of around $2.8 \mu \mathrm{G}$. In some experiments, cells were treated with $0.5 \mu \mathrm{M}$ OA (Calbiochem, Rome, Italy) or equal volumes of the solvent DMSO after $16 \mathrm{~h}$ of culture, and the incubation was continued for an additional $6 \mathrm{~h}$ to induce metaphase I entry. At the end of the incubation period, $1 \mathrm{ml}$ of the cell suspension was collected for morphological examination, and the remaining cells were collected by centrifugation at $1000 \mathrm{~g}$ for $10 \mathrm{~min}$ and immediately frozen at $-80^{\circ} \mathrm{C}$.

\section{Cytological methods}

Approximately $10^{6}$ spermatocytes, either after elutriation or following culture under unit gravity and microgravity conditions, were collected by centrifugation at $1000 \mathrm{~g}$ for $10 \mathrm{~min}$ at $4{ }^{\circ} \mathrm{C}$. Cell pellets were resuspended in hypotonic solution $(1 \mathrm{ml} 75 \mathrm{mM} \mathrm{KCl})$ and incubated for $20 \mathrm{~min}$ at $37^{\circ} \mathrm{C}$ to allow cell lysis. The nuclei suspensions obtained were fixed by adding $0.5 \mathrm{ml}$ methanol:acetic acid solution 
(3:1) and incubated for $20 \mathrm{~min}$ at $4{ }^{\circ} \mathrm{C}$. The suspensions were centrifuged for $20 \mathrm{~min}$ at 5000 r.p.m. at $4{ }^{\circ} \mathrm{C}$, and the pellets were washed three times with $1 \mathrm{ml}$ methanol:acetic acid solution (3:1). After the last wash, pellets were resuspended in $100 \mu \mathrm{l}$ methanol:acetic acid solution (3:1) and the suspension was dropped from 10 to $15 \mathrm{~cm}$ onto glass slides to allow spreading of the nuclei. Nuclei were stained with 5\% Giemsa (Sigma). Spermatocytes at middle-late pachytene stages and metaphase I were taken into account during the morphological examination by light microscopy. In some experiments, nuclei were stained with Hoechst (Sigma) and spermatocyte stages were scored by fluorescence microscopy. Each slide was independently analysed and quantified by three investigators, one of whom was not directly involved in the study.

\section{Immunoprecipitation experiments}

Control or treated spermatocytes (approximately $2 \times 10^{6}$ cell/sample) were homogenized in lysis buffer $(50 \mathrm{mM}$ Hepes, pH 7.5, $100 \mathrm{mM} \mathrm{NaCl}, 50 \mathrm{mM} \beta$-glycerophosphate, $15 \mathrm{mM}$ EGTA, $15 \mathrm{mM} \mathrm{MgCl} 2,0.1 \mathrm{mM}$ sodium orthovanadate, $1 \mathrm{mM}$ dithiothreitol (DTT), $10 \mu \mathrm{g} / \mathrm{ml}$ leupeptin, $10 \mu \mathrm{g} / \mathrm{ml}$ aprotinin and $1 \mathrm{mM}$ phenylmethylsulphonyl fluoride) and incubated for $10 \mathrm{~min}$ on ice. Cell lysates were then centrifuged for $10 \mathrm{~min}$ at $10000 \mathrm{~g}$, the supernatants were collected and protein concentration was determined (Bradford 1976). In some experiments, aliquots of the lysate were directly used for MAPK assay and, in most cases, aliquots containing $200 \mu \mathrm{g}$ proteins were used for immunoprecipitation. Protein A/G-Sepharose beads (Sigma) were preadsorbed to $1 \mu \mathrm{g}$ rabbit polyclonal antiErk1 (Santa Cruz Biotechnology, Santa Cruz, CA, USA), goat polyclonal anti-p90Rsk2 (Santa Cruz) or mouse monoclonal anti-cyclin B1 (Santa Cruz) antibodies for $60 \mathrm{~min}$ in phosphate-buffered saline containing $0.05 \% \mathrm{BSA}$ under constant shaking at $4{ }^{\circ} \mathrm{C}$. At the end of the incubation, the beads were washed twice with lysis buffer, and then incubated for $90 \mathrm{~min}$ at $4{ }^{\circ} \mathrm{C}$ with the spermatocyte extracts $(500 \mu \mathrm{g}$ protein) under constant shaking. Sepharose bead-bound immunocomplexes were rinsed three times with lysis buffer and washed twice with the appropriate kinase buffer for immunokinase assays (see below).

\section{Immunokinase assays}

Immunocomplexes bound to Sepharose beads obtained from immunoprecipitation of cell extracts were rinsed twice with kinase buffer (50 mM Hepes, pH 7.5, $5 \mathrm{mM} \beta$ glicerophosphate, $2 \mathrm{mM}$ EGTA, $15 \mathrm{mM} \mathrm{MgCl}, 0.1 \mathrm{mM}$ sodium orthovanadate, $1 \mathrm{mM}$ DTT, $10 \mu \mathrm{g} / \mathrm{ml}$ leupeptin and $10 \mu \mathrm{g} / \mathrm{ml}$ aprotinin). Kinase reactions were carried out in $40 \mu \mathrm{l}$ for $30 \mathrm{~min}$ at $30^{\circ} \mathrm{C}$ under constant shaking in kinase buffer supplemented with $10 \mu \mathrm{M}{ }^{32} \mathrm{P}-\gamma$-ATP $(0.2 \mu \mathrm{Ci} / \mu \mathrm{l})$, $1 \mu \mathrm{g}$ cAMP-dependent protein kinase inhibitor and the appropriate substrate $(0.5 \mathrm{mM}$ myelin basic protein (MBP)derived peptide (Santa Cruz) for Erk1; $100 \mu$ M S6 peptide (Calbiochem) for p90Rsk2; $1 \mu \mathrm{g}$ histone $(\mathrm{H}) 1$, type III-S
(Sigma) for MPF). At the end of the incubation, kinases bound to the beads were separated by centrifugation and supernatants were spotted onto P81 phosphocellulose paper squares (Whatman, Schleicher and Schuell, Keene, $\mathrm{NH}$, USA) and immersed immediately in $0.1 \%$ phosphoric acid. Paper squares were washed five times for $10 \mathrm{~min}$ and air-dried. The incorporated radioactivity was determined by scintillation counting. In some experiments, kinase assays were performed directly in spermatocyte soluble extract using $2 \mu \mathrm{g}$ proteins, according to the same experimental procedure.

\section{Western blot}

Spermatocytes cell extracts $(20 \mu \mathrm{g})$ were analyzed by Western blot using the rabbit anti-Erk1 antibody (Santa Cruz) or mouse anti- $\beta$-tubulin (Sigma) at 1:1000 dilution and chemiluminescence detection (Santa Cruz) as previously described (Sette et al. 1999).

\section{Results}

The spermatocyte fraction obtained by elutriation used in our experiments was enriched in cells at middle-late pachytene stages $(85-90 \%)$, and the $10-15 \%$ contaminating cells were spermatogonia, early meiotic cells, somatic cells and rare round spermatids. Figure 1 shows the morphological characteristics of the Giemsa-stained spermatocyte nuclei used as the criteria in scoring midpachytene (Fig. 1a), late-pachytene (Fig. 1b) and metaphases (Fig. 1c) in the spermatocyte-enriched cell fraction. Figure 1 also shows representative fields of nuclear squashes obtained from spermatocytes that were cultured for $24 \mathrm{~h}$ either at unit gravity in culture dishes (Fig. 1d) or under microgravity in RCCS (Fig. 1e). An increase in the number of late-pachytene spermatocytes was evident in the cell population kept under microgravity conditions with respect to the population cultured at unit gravity. However, we were unable to detect an increase in the metaphase of the first meiotic division following culture in RCCS, suggesting that cells do not reach this stage in the culture period examined. Quantitative analysis of cell populations following culture in the two conditions was performed by counting 500 nuclei from each sample, and calculating the mean values \pm s.D. for the different pachytene stages (Fig. 2). In simulated microgravity, an approximate $20 \%$ reduction of spermatocytes at the mid-pachytene stage could be observed with respect to cultures at unit gravity. This reduction was due to a shift to the late-pachytene stage, which is increased proportionally under microgravity, hence doubling the relative amount of this pachytene stage with respect to unit gravity.

\section{Specific activation of MAPK in mouse spermatocytes under microgravity conditions}

It was previously shown that MAPKs were activated during the meiotic transition of mouse pachytene spermatocytes induced by stimulation with $\mathrm{OA}$ and that this activation 


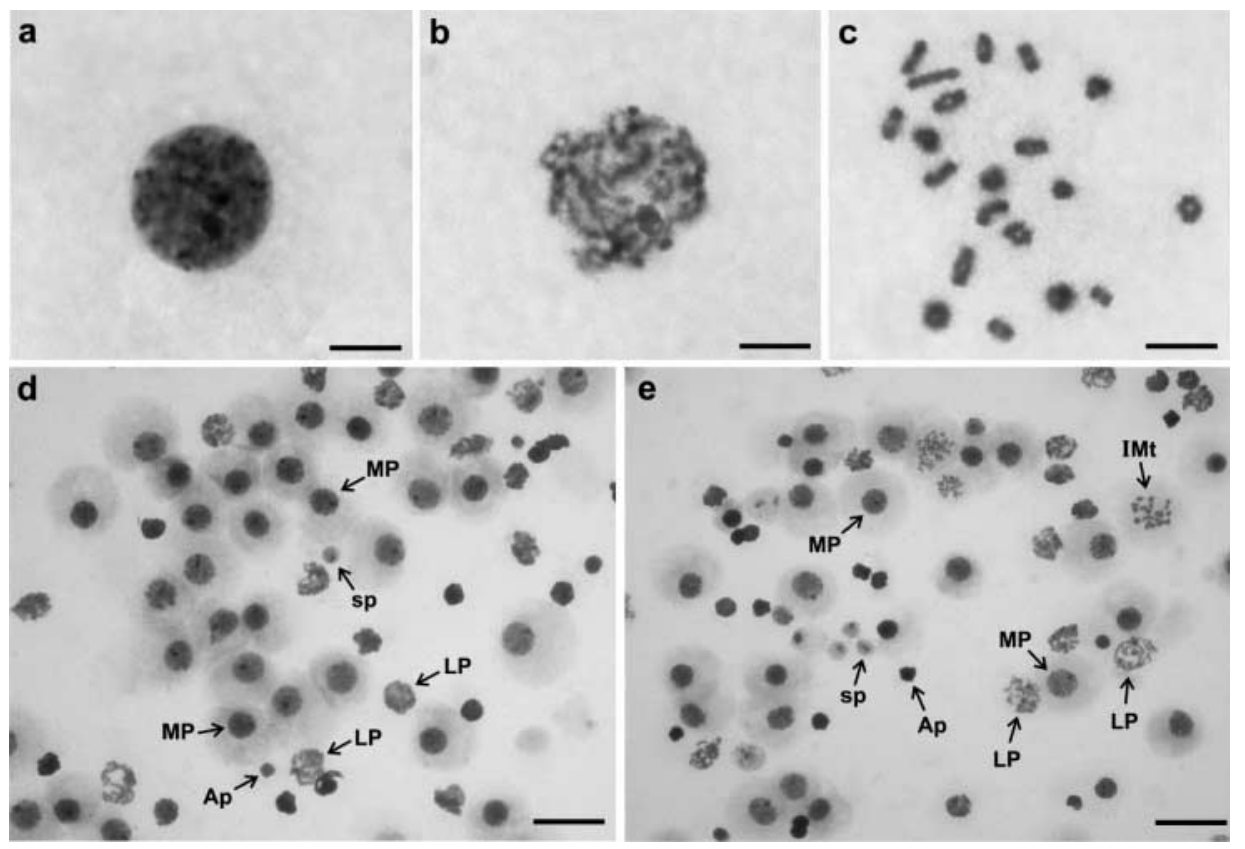

Figure 1 Morphological characteristics of germ cell fractions enriched in middle-late pachytene spermatocyte cultured under unit gravity or microgravity condition. The morphological criteria used for identifying Giemsa-stained nuclei are exemplified in (a) mid-pachytene, (b) latepachytene and (c) I meiotic metaphase $(100 \times$ objective). ( $d$ and $\mathrm{e})$ Representative fields of nuclear preparations following cell culture at (d) unit gravity and (e) microgravity in RCCS $(20 \times$ objective). Arrows indicate mid-pachytene (MP), latepachytene (LP), first meiosis metaphases (IMt), apoptotic and/or degenerating cells (Ap) and round spermatids (sp). Bars $=10 \mu \mathrm{m}(\mathrm{a}, \mathrm{b}$ and $\mathrm{c}$ ) and $80 \mu \mathrm{m}$ ( $\mathrm{d}$ and e). Experimental details are described in Materials and Methods. was required for efficient condensation of the chromatin (Sette et al. 1999, Di Agostino et al. 2002). To test if the meiotic progression obtained by culturing mouse spermatocytes in RCCS was also accompanied by MAPK activation, we performed a kinase assay using a peptide derived from MBP as a specific MAPK substrate and total soluble cell extracts. MAPK activity was increased 3.5fold in cells cultured under microgravity with respect to unit gravity (Fig. 3A). Western blot analysis demonstrated that the samples contained equal amount of Erks in the soluble extracts (Fig. 3B).

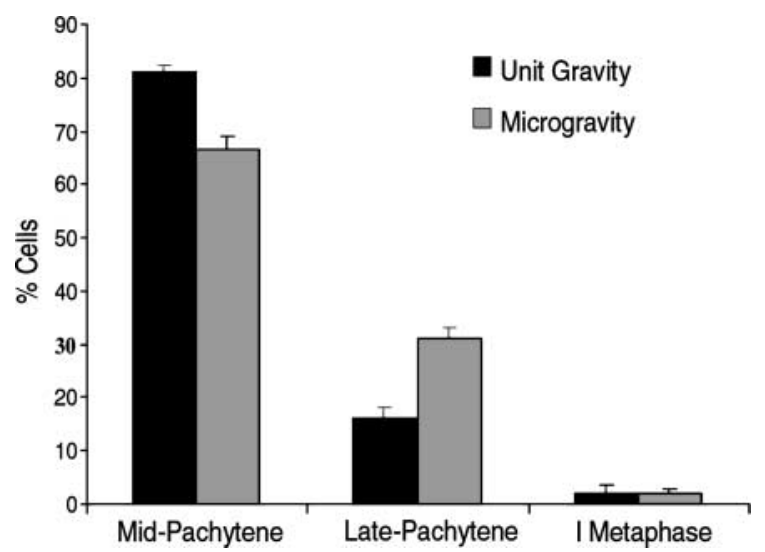

Figure 2 Quantitative data on the progression of mid-pachytene spermatocyte to the late-pachytene stage under simulated microgravity. At least 500 Giemsa-stained nuclei were counted in each squash from cell suspensions cultured for $24 \mathrm{~h}$ at unit gravity (solid bars) or under microgravity (shaded bars). The values \pm S.D. reported in the figure are relative only to pachytene spermatocytes, whereas the 10 $15 \%$ contaminating cells present in the fraction were not taken into account. The data are the average \pm s.D. from three separate experiments.
To investigate whether the increase in MAPK activity could be attributed to Erk1, as was previously shown with OA-induced meiotic progression (Sette et al. 1999), we evaluated the phosphorylative activity of immunoprecipitates obtained with anti-Erk1 antibodies from extracts of spermatocytes cultured in unit gravity and microgravity. As observed for total MAPK activity in soluble extracts,
A

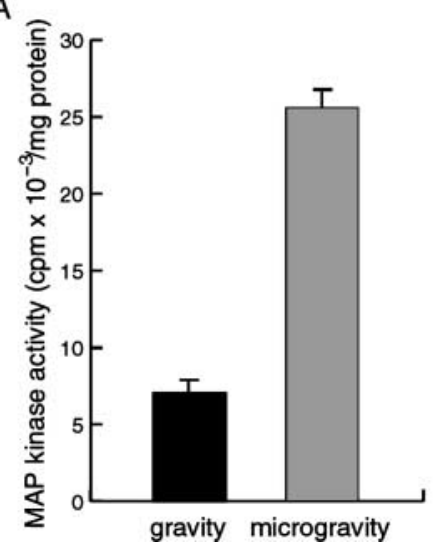

B

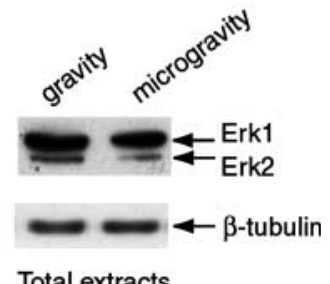

Figure 3 Microgravity induces MAPK activation in pachytene spermatocytes. (A) Kinase assay was performed by adding $2 \mu \mathrm{g}$ cell lysate to $40 \mu \mathrm{l}$ kinase buffer containing $1 \mu \mathrm{g}$ cAMP-dependent protein kinase inhibitor, $10 \mu \mathrm{M}{ }^{32} \mathrm{P}$ - $\gamma$-ATP and $0.5 \mathrm{mM}$ MBP-derived peptide as specific Erk1 substrate. The reaction was spotted onto p81 phosphocellulose filters and incorporated radioactivity was determined by scintillation counting following washes in $0.1 \%$ phosphoric acid (see Materials and Methods for experimental details). (B) Western blot analysis of cell lysates $(20 \mu \mathrm{g})$ with anti-Erk1 (upper panel) and anti$\beta$-tubulin (lower panel) antibodies shows that equal amounts of kinase were present in the lysates. The arrows indicate the position of $\beta$-tubulin and the $44 \mathrm{kDa}$ Erk1 and a faint band corresponding to the homologous $42 \mathrm{kDa}$ Erk2, also recognized by the anti-Erk1 antibody. 
the microgravity culture condition stimulated the activity of immunoprecipitated Erk1, suggesting that this isoform is the preponderant MAPK activated during the meiotic progression caused by this culture treatment (Fig. 4A).

\section{p90Rsk2 is activated in spermatocytes by microgravity}

The p90Rsk2 kinase acts downstream of Erk1 in mouse spermatocytes in the pathway that leads to chromatin condensation during OA-induced $\mathrm{G} 2 / \mathrm{M}$ progression (Di Agostino et al. 2002). To determine if activation of Erk1 under RCCS condition also caused activation of p90Rsk2, we immunoprecipitated p90Rsk2 from extracts of spermatocytes cultured either under gravity or microgravity conditions and assayed its activity using $\mathrm{S} 6$ peptide as substrate. As shown in Fig. 4B, a significant increase in p90Rsk2 activity was observed in spermatocytes cultured under microgravity. This result indicated that a complete activation of the MAPK/p90Rsk2 pathway is achieved by culturing mouse spermatocytes under microgravity conditions.

\section{Effect of microgravity on activation of MPF in pachytene spermatocytes}

A common feature of both meiotic and mitotic G2/M progression is the activation of MPF. To determine if meiotic progression induced by microgravity conditions affected MPF activity in mouse spermatocytes, we immunoprecipitated MPF from spermatocyte extracts using a monoclonal anti-cyclin B1 antibody, which immunoprecipitates most of the MPF activity in these cells (Chapman \& Wolgemuth 1994, Sette et al. 1999). The activity of the immunoprecipitated kinase was assayed using $\mathrm{H} 1$ histone as substrate. The results shown in Fig. $4 \mathrm{C}$ indicate that the microgravity condition is able to induce activation of MPF (approximately 2-fold), confirming the previous observation on meiotic progression of mouse spermatocytes induced by this cell culture condition.

\section{Discussion}

In this work, we have demonstrated that culturing mouse spermatocytes under microgravity conditions in a RCCS allows their progression through the pachytene stage of the first meiotic prophase. The cell population used in the present study was represented by mid-late pachytene spermatocytes and minor contaminants $(10-15 \%$ total) of somatic cells, spermatogonia and early meiotic cells, and rare round spermatids. After $24 \mathrm{~h}$ of culture in simulated microgravity conditions in a RCCS, we observed a significant increase in the number of spermatocyte nuclei with highly condensed chromatin which we identified as latepachytene spermatocytes. This increase was accompanied by a parallel reduction of nuclei with less condensed chromatin identified as mid-pachytene spermatocytes. No such progression along meiotic prophase could be observed in cultures at unit gravity, unless spermatocytes are treated with OA as previously demonstrated (Wiltshire et al. 1995).

The effects of $\mathrm{OA}$ on the cell cycle are mediated by inhibition of serine/threonine phosphatase $2 \mathrm{~A}$ with the consequent activation of several kinases that are under the negative control of this phosphatase. Among them, the cdc2 kinase (Felix et al. 1990) and MAPKs (Anderson et al. 1990, Casillas et al. 1993, Alessi et al. 1995) have been shown to play a role during the meiotic progression induced by the treatment of mouse spermatocytes with OA (Wiltshire et al. 1995, Sette et al. 1999). The pharmacological effect of OA treatment for $4-6 \mathrm{~h}$ causes a complete G2/M transition with full condensation of
A

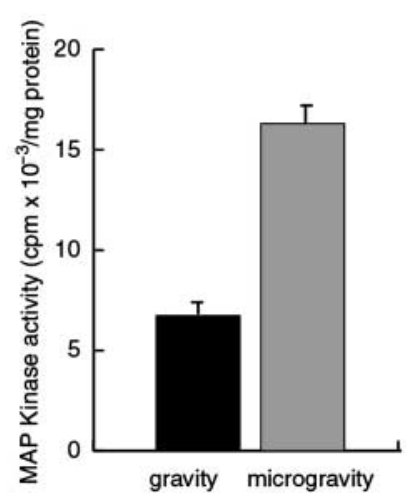

B

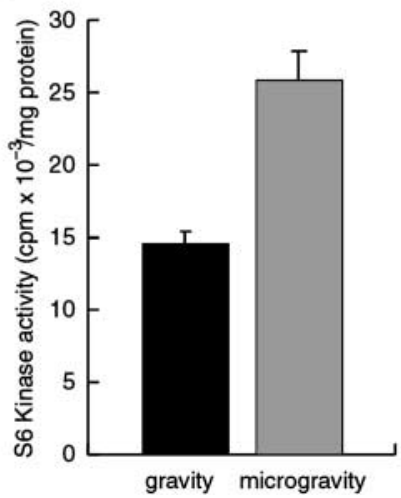

C

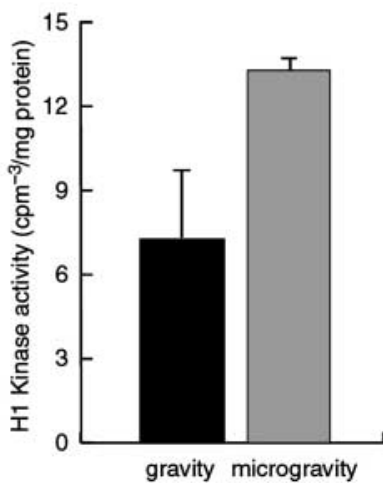

Figure 4 Erk1, p90Rsk2 and MPF are activated under microgravity conditions in pachytene spermatocytes. Kinase activity was measured following immunoprecipitation of spermatocyte extracts with proteinA/G-Sepharose beads preadsorbed with (A) rabbit polyclonal anti-Erk1, (B) goat polyclonal anti-p90Rsk2 or (C) mouse monoclonal anti-cyclin B1. Sepharose bead-bound immunocomplexes were used as the enzyme source in the kinase assay using ${ }^{32} \mathrm{P}-\gamma$-ATP as phosphate donor and (A) MBP-derived peptide as substrate for Erk1, (B) S6 peptide as substrate for p90Rsk2 or (C) $\mathrm{H} 1$ as substrate for MPF. The incorporated radioactivity was measured by spotting the reaction supernatant on phosphocellulose paper. See Materials and Methods for experimental details. The values are the average \pm s.D. of the results from three separate experiments. 
chromatin to form chromosomes in almost $80-90 \%$ of mid-late pachytene spermatocytes in culture. By contrast, we observed that the spontaneous progression to latepachytene obtained under microgravity conditions affected a smaller percentage of the cell population (approximately $30 \%$ ). It is possible that the activation of several kinase pathways by $\mathrm{OA}$ is able to relieve the control of several meiotic checkpoints that normally delay the prophase of the first meiotic division. Indeed, both the DNA damage and the spindle assembly checkpoints are governed by the activity of protein kinases and phosphatases, which are likely affected by long-term incubation with OA. On the other hand, we hypothesized that RCCS conditions allow a physiological progression to more advanced stages of the meiotic prophase. Since progression through this stage in vivo takes several days, the nuclear morphology changes very little before reaching advanced stages where final chromosome condensation occurs (Cobb \& Handel 1998, Wiltshire et al. 1998). Thus, our observation that only a small percentage of cells shifts from mid- to late-pachytene under microgravity conditions can be explained by the following considerations: since the spermatocyte population that we isolated represents a mixture of pachytene stages that are at least 5 days apart from the earliest to the more advanced form, during the $24 \mathrm{~h}$ of our experiment we might have the morphological signal of progression only for that group of spermatocytes that develop a higher chromatin condensation (apparently one-fifth of the mid-pachytene population), even though each represented pachytene stage might be progressing. In support of our interpretation is the observation that molecular parameters that accompany chromosome condensation (i.e. MAPK and MPF activity) show a more dramatic change than the morphological ones and are not observed in cultures at unit gravity.

We have previously demonstrated that activation of the MAPK Erk1 is required for the efficient chromatin condensation during the first G2/M transition induced by OA in mouse spermatocytes (Sette et al. 1999). Moreover, activation of the MAPK pathway triggered the activation of Nek2 (Di Agostino et al. 2002), a serine/threonine kinase that is associated with meiotic chromatin (Rhee \& Wolgemuth 1997). More recently, we have shown that Nek2 phosphorylates the chromatin architectural protein Hmga2, causing its release from the condensing chromatin (Di Agostino et al. 2004). However, direct evidence that activation of the MAPK pathway occurs naturally in mouse spermatocytes not exposed to OA was still lacking. The study described here has demonstrated that activation of Erk1 and its effector p90Rsk2 accompany the spontaneous progression allowed by culturing mouse spermatocyte in RCCS conditions, suggesting that MAPK activation is not just a side-effect of stimulation with the phosphatase inhibitor. In agreement with this hypothesis is the observation that a prophase stage-dependent MAPK activation also seems to occur in mouse fetal oocytes (M De Felici, personal communication).
OA stimulation of pachytene spermatocytes leads to a rapid progression into metaphase. This progression is notably accelerated as compared with the in vivo situation $(6 \mathrm{~h}$ compared with days) and terminates with an arrest at metaphase. This arrest is probably due to disassembly of the meiotic spindle caused by OA treatment (ZernickaGoetz \& Maro 1993), as suggested by the observation that the meiotic chromosomes thus obtained are not aligned on the metaphase spindle but rather clustered at the periphery of the nuclear matrix (data not shown). Nevertheless, metaphase entry is confirmed by the morphology of the chromosomes and by the 4- to 5-fold activation of MPF observed in OA-treated spermatocytes (Wiltshire et al. 1995). We report that, under simulated microgravity conditions, MAPK activation in the mouse spermatocyte reaches the same level as that obtained with OA treatment (4-fold), while MPF activity reaches only a 2-fold increase which is not reflected, on the other hand, by a significant increase in metaphase entry. Since it is possible that MAPK activation occurs at an earlier meiotic stage with respect to activation of MPF, which is maximal at metaphase, RCCS conditions appear to promote a slower and less dramatic progression of meiotic prophase with respect to that caused by OA treatment, and the timing of this progression resembles more closely that of the physiological prophase in the seminiferous tubule.

The RCCS seems to be an important tool to dissect the direct effect of microgravity on isolated male germ cells at different stage of differentiation from the effect of microgravity on spermatogenesis in vivo. Several studies performed either with experiments in satellites (Philpott et al. 1985, Sapp et al. 1990, Amann et al. 1992) or with hindlimb-suspended rats to simulate microgravity (Tash et al. 2002) have described a detrimental effect of microgravity on spermatogenesis. Our observation of a positive effect of microgravity on meiotic progression, even though limited to a single event of spermatogenesis, would strengthen the hypothesis that altered physiological parameters, like reduced testosterone levels, increased cortisol concentration, reduced blood circulation or increased temperature at the level of the testis, rather than a direct effect on germ cells, are the cause of the altered spermatogenesis (Tash et al. 2002).

Enzymatic activation under microgravity has also been described at the kinetic level on isolated lipoxygenase-1 in vitro (Maccarrone et al. 2001). Moreover, an increase in serine phosphorylation of the sea urchin sperm flagellar protein FP130 has been observed in vivo in experiments conducted in space (Tash \& Bracho 1999). Although a clear interpretation of the effect of microgravity on enzyme activities has not been provided, experiments performed with $\mathrm{D}_{2} \mathrm{O}$ suggest that a change in the properties of water occurring under microgravity might alter signal transduction and enzyme activation (Tash \& Bracho 1999). Clearly, further experiments are required to elucidate the effects of microgravity on both whole cells and isolated enzymes. 
In conclusion, our work has suggested that culturing mouse spermatocytes under microgravity conditions might be helpful to study meiotic transitions, both biochemically and morphologically, without the dramatic effects on the homeostasis of phosphorylation/dephosphorylation events caused by treatment with OA.

\section{Acknowledgements}

We thank Professor Massimo De Felici for personal communication of unpublished results, and for critical reading of the manuscript. This work was supported by a grant from the Agenzia Spaziale Italiana (ASI) and from the Centro d'Eccellenza per lo Studio del Rischio Genomico in Patologie Complesse.

\section{References}

Abrieu A, Doree M \& Fisher D 2001 The interplay between cyclin-BCdc2 kinase (MPF) and MAP kinase during maturation of oocytes. Journal of Cell Science 114 257-267.

Alessi DR, Cuenda A, Cohen P, Dudley DT \& Saltiel AR 1995 PD 098059 is a specific inhibitor of the activation of mitogen-activated protein kinase kinase in vitro and in vivo. Journal of Biological Chemistry $27027489-27494$.

Amann RP, Deaver DR, Zirkin BR, Grills GS, Sapp WJ, Veeramachaneni DN, Clemens JW, Banerjee SD, Folmer J \& Gruppi CM et al. 1992 Effects of microgravity or simulated launch on testicular function in rats. Journal of Applied Physiology 73 174S-185S.

Anderson NG, Maller JL, Tonks NK \& Sturgill TW 1990 Requirement for integration of signals from two distinct phosphorylation pathways for activation of MAP kinase. Nature 343 651-653.

Bradford MM 1976 A rapid and sensitive method for the quantitation of microgram quantities of protein utilizing the principle of protein-dye binding. Analytical Biochemistry 72 248-254.

Casillas AM, Amaral K, Chegini-Farahani S \& Nel AE 1993 Okadaic acid activates p42 mitogen-activated protein kinase (MAP kinase; ERK-2) in B-lymphocytes but inhibits rather than augments cellular proliferation: contrast with phorbol 12-myristate 13-acetate. Biochemical Journal 290 545-550.

Chapman DL \& Wolgemuth DJ 1994 Regulation of M-phase promoting factor activity during development of mouse male germ cells. Developmental Biology 165 500-506.

Cobb J \& Handel MA 1998 Dynamics of meiotic prophase I during spermatogenesis: from pairing to division. Seminars in Cell Developmental Biology 9 445-450.

Colledge WH, Carlton MB, Udy GB \& Evans MJ 1994 Disruption of C-mos causes parthenogenetic development of unfertilized mouse eggs. Nature 370 65-68.

Di Agostino S, Rossi P, Geremia R \& Sette C 2002 The MAPK pathway triggers activation of Nek2 during chromosome condensation in mouse spermatocytes. Development 129 1715-1727.

Di Agostino S, Fedele M, Chieffi P, Fusco A, Geremia R, Rossi P \& Sette C 2004 Phosphorylation of high mobility group protein A2 by Nek2 kinase during the first meiotic division in mouse spermatocytes. Molecular Biology of the Cell 15 1224-1232.

Dupre A, Jessus C, Ozon R \& Haccard O 2002 Mos is not required for the initiation of meiotic maturation in Xenopus oocytes. EMBO Journal 21 4026-4036.

Felix MA, Labbe JC, Doree M, Hunt T \& Karsenti E 1990 Triggering of cyclin degradation in interphase extracts of amphibian eggs by cdc2 kinase. Nature 346 379-382.

Gross SD, Schwab MS, Taieb FE, Lewellyn AL, Qian YW \& Maller JL 2000 The critical role of the MAP kinase pathway in meiosis II in
Xenopus oocytes is mediated by p90(Rsk). Current Biology 10 430-438.

Hashimoto N, Watanabe N, Furuta $Y$, Tamemoto $H$, Sagata $N$, Yokoyama M, Okazaki K, Nagayoshi M, Takeda N \& Ikawa Y et al. 1994 Parthenogenetic activation of oocytes in c-mos-deficient mice. Nature 370 68-71.

Ingram M, Techy GB, Saroufeem R, Yazan O, Narayan KS, Goodwin TJ \& Spaulding GF 1997 Three-dimensional growth patterns of various human tumor cell lines in simulated microgravity of a NASA bioreactor. In Vitro Cellular and Developmental Biology. Animals 33 459-466.

Nakamura K, Kuga H, Morisaki T, Baba E, Sato N, Mizumoto K, Sueishi K, Tanaka M \& Datano M 2002 Simulated microgravity culture system for a 3-D carcinoma tissue model. Biotechniques 33 1068-1070.

Maccarrone M, Bari M, Battista N \& Finazzi-Agrò A 2001 The catalytic efficiency of soybean lipoxygenase- 1 is enhanced at low gravity. Biophysical Chemistry 90 303-306.

Maller JL, Gross SD, Schwab MS, Finkielstein CV, Taieb FE \& Qian YW 2001 Cell cycle transitions in early Xenopus development. Novartis Foundation Symposium 237 58-73.

Meistrich ML 1977 Separation of spermatogenic cells and nuclei from rodent testes. Methods in Cell Biology 15 15-54.

Palmer A, Gavin AC \& Nebreda AR 1998 A link between MAP kinase and p34(cdc2)/cyclin B during oocyte maturation: p90(rsk) phosphorylates and inactivates the p34(cdc2) inhibitory kinase Myt1. EMBO Journal 17 5037-5047.

Petronczki M, Siomos MF \& Nasmyth K 2003 Un menage à quatre: the molecular biology of chromosome segregation in meiosis. Cell 112 423-440.

Philpott DE, Sapp W, Williams C, Stevenson J, Black S \& Corbett R 1985 Reduction of the spermatogonial population in rat testes flown on Space Lab-3. Physiologist 28 S211-S212.

Qian YW, Erikson E, Taieb FE \& Maller JL 2001 The polo-like kinase plx1 is required for activation of the phosphatase cdc25c and cyclin b-cdc2 in Xenopus oocytes. Molecular Biology of the Cell $121791-1799$.

Rhee K \& Wolgemuth DJ 1997 The NIMA-related kinase 2. Nek2, is expressed in specific stages of the meiotic cell cycle and associates with meiotic chromosomes. Development 124 2167-2177.

Roeder GS 1997 Meiotic chromosomes: it takes two to tango. Genes and Development 11 2600-2621.

Sagata N 1997 What does Mos do in oocytes and somatic cells? Bioessays 19 13-21.

Sagata N, Oskarsson M, Copeland T, Brumbaugh J \& Vande Woude GF 1988 Function of c-mos proto-oncogene product in meiotic maturation in Xenopus oocytes. Nature 335 519-525.

Sagata N, Daar I, Oskarsson M, Showalter SD \& Vande Woude GF 1989 The product of the mos proto-oncogene as a candidate 'initiator' for oocyte maturation. Science 245 643-646.

Sapp WJ, Philpott DE, Williams CS, Kato K, Stevenson J, Vasquez M \& Serova LV 1990 Effects of the spaceflight on the spermatogonial population of rat seminiferous epithelium. FASEB Journal $\mathbf{4}$ 101-104.

Sassa T, Richter WW, Uda N, Suganuma M, Suguri H, Yoshizawa S, Hirota M \& Fujiki H 1989 Apparent 'activation' of protein kinases by okadaic acid class tumor promoters. Biochemical and Biophysical Research Communications 159 939-944.

Sette C, Barchi M, Bianchini A, Conti M, Rossi P \& Geremia R 1999 Activation of the mitogen-activated protein kinase Erk1 during meiotic progression of mouse pachytene spermatocytes. Journal of Biological Chemistry 274 33571-33579.

Tash JS \& Bracho GE 1999 Microgravity alters protein phosphorylation changes during initiation of sea urchin sperm motility. FASEB Journal 13 S43-S54.

Tash JS, Johnson DC \& Enders GC 2002 Long-term ( 6 wk) hindlimb suspension inhibits spermatogenesis in adult male rats. Journal of Applied Physiology 92 1191-1198. 
Verlhac MH, Kubiak JZ, Weber M, Geraud G, Colledge WH, Evans MJ \& Maro B 1996 Mos is required for MAP kinase activation and is involved in microtubule organization during meiotic maturation in the mouse. Development 122 815-822.

Verlhac MH, Lefebvre C, Guillaud P, Rassinier P \& Maro B 2000 Asymmetric division in mouse oocytes: with or without Mos. Current Biology 10 1303-1306.

Wiltshire T, Park C, Caldwell KA \& Handel MA 1995 Induced premature G2/M-phase transition in pachytene spermatocytes includes events unique to meiosis. Developmental Biology 169 $557-567$.
Wiltshire T, Park C \& Handel MA 1998 Chromatin configuration during meiosis I prophase of spermatogenesis. Molecular Reproduction and Development 49 70-80.

Zernicka-Goetz M \& Maro B 1993 Okadaic acid affects spindle organization in metaphase II-arrested rat oocytes. Experimental Cell Research 207 189-193.

Received 27 January 2004

First decision 31 March 2004

Accepted 26 April 2004 